\title{
Effect of mobile text message reminders on routine childhood vaccination: a systematic review and meta-analysis
}

Zeleke Abebaw Mekonnen ${ }^{1 *}$, Kassahun Alemu Gelaye ${ }^{2}$, Martin C. Were ${ }^{3}$, Kassahun Dessie Gashu ${ }^{1}$ and Binyam Chakilu Tilahun ${ }^{1}$

\begin{abstract}
Background: The World Health Organization estimates that 29\% of under-five mortality could be prevented with existing vaccines. However, non-consistent attendance for immunization appointments remains a global challenge to healthcare providers. Thus, innovative strategies are required to reach the last mile where technology could be effectively utilized to achieve better compliance with children immunization schedules. Therefore, the aim of the review was to systematically collect and summarize the available evidence on the effectiveness of text message reminders on childhood vaccination.
\end{abstract}

Methods: This review was conducted according to a priori published protocol on PROSPERO. A systematic literature search of databases (PubMed/MEDLINE, EMBASE, Cochrane/Wiley library, and Science direct) was conducted. Eligibility and risk of bias assessments were performed independently by two reviewers. PRISMA flow diagrams were used to summarize the study selection process. Taking into account the level of heterogeneity, a random effects model was used and risk ratios with their $95 \% \mathrm{Cl}$ were used to present the pooled estimates. To investigate the sources of heterogeneity, subgroup analysis and meta-regression analysis were also considered. In this review, publication bias was assessed statistically using Harbord test.

Results: A total of 1771 articles were searched. Out of those 1771 articles, 558 duplicated articles were removed. About 1213 articles were further screened, and finally, ten articles met the inclusion criteria. The meta-analysis showed that there is a significant effect of text message reminders on childhood vaccination coverage ( $\mathrm{RR}=1.11 ; 95 \% \mathrm{Cl} 1.05-1.17)$ with a moderate level of heterogeneity $\left(R^{2}=64.3 \%, P=0.003\right)$. The results from the Harbord test suggested that there is no evidence for publication bias $(P=0.340)$.

Conclusion: This review highlights the potential benefits of incorporating mobile text message reminders into the standard management of childhood immunizations, especially in low- and middle-income countries. The frequency and timing of the text message reminders are also crucial in determining the effectiveness of text message reminders. Hence, mHealth interventions deserve more attention as a potential innovation to improve healthcare programs.

Systematic review registration: PROSPERO CRD42017074230

Keywords: Vaccination, Immunization, Text message, mHealth

\footnotetext{
* Correspondence: zelekeabebaw7@gmail.com

'Department of Health Informatics, Institute of Public Health, College of

Medicine and Health Sciences, University of Gondar, Gondar, Ethiopia

Full list of author information is available at the end of the article
}

(c) The Author(s). 2019 Open Access This article is distributed under the terms of the Creative Commons Attribution 4.0 International License (http://creativecommons.org/licenses/by/4.0/), which permits unrestricted use, distribution, and reproduction in any medium, provided you give appropriate credit to the original author(s) and the source, provide a link to the Creative Commons license, and indicate if changes were made. The Creative Commons Public Domain Dedication waiver (http://creativecommons.org/publicdomain/zero/1.0/) applies to the data made available in this article, unless otherwise stated. 


\section{Background}

Vaccinations are widely regarded as one of the most costeffective public health interventions that help to reduce global child morbidity and mortality. The World Health Organization (WHO) estimates that 29\% of under-five mortality could be prevented with existing vaccines [1]. However, vaccine-preventable diseases are still a major cause of morbidity and mortality worldwide, especially in low- and middle-income countries (LMICs) [2].

In 2016, global vaccination coverage has stalled at $86 \%$ with an estimated 19.5 million infants worldwide not reached with routine immunization services. As a consequence of this continued failure to reach optimal immunization coverage, 1.5 million children die each year from vaccine-preventable diseases [1].

This high prevalence of childhood vaccine-preventable diseases can be significantly reduced through adherence to confirmed vaccination schedules. However, many barriers to vaccination compliance exist and the proportion of children who had not completed the vaccination program ranged from 23.3 to $76.3 \%$ in developing countries [3-5]. The main parental barriers to vaccination include confusion and difficulty in tracking vaccination schedules, low parent knowledge about the benefits of vaccination, missing due dates, and fear of vaccinations' complications [6-8].

A study in India found the reasons for infants missing vaccination are due to prior reminders not given (32.9\%) and parent's forgetfulness (26.6\%) [6] indicating that compliance to the recommended vaccination schedule is a challenge for healthcare systems. Thus, innovative strategies are urgently required where technology could be effectively utilized to achieve better compliance with children immunization schedules.

The increased penetration of Information and Communications Technology (ICT) in health care offers an opportunity to strengthen the health systems [9, 10]. Among the different ICT interventions, mobile phone-based (mHealth) text messaging has gained popularity and may be the key to penetrating hard to reach populations. Text message reminders provide a cost-effective method of relaying health information and reminders [11, 12]. Evidence also showed that these demand-side interventions $[13,14]$ target characteristics such as forgetfulness in reducing dropouts from vaccination [15-17].

\section{Justification for the review}

Globally, non-attendance for immunization appointments remains a challenge to healthcare providers. In many countries, immunization coverage is low [1], routine immunization systems are weak, and the awareness of the community about the immunization program is low $[2,18]$. Though different strategies have been tried to increase the number of children vaccinated, there is a continued failure to achieve vaccination targets $[1,10]$.

Making well-informed decisions about how best to achieve and sustain high and equitable immunization coverage will depend partly on decision makers accessing the best scientific evidence about what interventions work and integrating this evidence into existing health systems. For any intervention to be adopted in a setting, it must be designed to meet the particular needs of the setting and in the magnitude that best addresses the needs. Although mHealth initiatives are found to be important in health care, its effectiveness varies with implementation context. Therefore, the increased drive to develop and scale-up mHealth interventions with the dynamic ICT environment demands the availability of robust and current evidence.

We set out to explore the evidence around the use of text messaging to improve childhood vaccination, and to our knowledge, found no published systematic review and meta-analysis so far on this topic to help guide decisionmaking. Therefore, this review would summarize the available evidence on the effectiveness of text message reminders as compared to standard care on childhood vaccination for further public health interventions.

\section{Methods}

\section{Protocol and registration}

This review was conducted according to an a priori published protocol on PROSPERO International Prospective Register of systematic reviews for publication registration number CRD42017074230 (available at https://www.crd. york.ac.uk/PROSPERO/\#myprospero).

\section{Eligibility criteria}

PICOS approach was used to set inclusion and exclusion criteria.

\section{Inclusion criteria}

1. Type of studies: randomized controlled trial studies

2. Types of participants: caregivers/parents of children who are under 5 years of age

3. Types of interventions: we include interventions in which mobile phone text messages provide reminders related to vaccinations

4. Types of controls: studies with comparison group of routine/standard care

5. Types of outcome measures: studies will be eligible for inclusion if they reported vaccination coverages for one of the vaccines or all

\section{Exclusion criteria}

Studies with no accessible full text, not in English language, and studies which do not report specific vaccination outcomes quantitatively 


\section{Information sources and search strategy}

Initially, databases were searched for the same systematic review done before to avoid duplications of efforts. Search terms were pre-defined for a comprehensive search strategy that included text fields within records, and Medical Subject Headings (MeSH terms) were used to expand the searching. We used Boolean operators for the search strategies. A systematic literature search of databases (PubMed/MEDLINE, EMBASE, Cochrane/Wiley library, and Science direct) was conducted. Gray literatures were searched using Google and Google Scholar. In addition, reference lists of relevant studies were identified and reviewed for inclusion (Table 1).

\section{Study selection and data collection}

Database search results were combined, and duplicate articles were removed using Endnote (version7) and manually. Eligibility assessment was performed independently by two reviewers (Zeleke Abebaw and Kasahun Dessie). Discrepancies between the two reviewers in this process were discussed with the other review team member (Binyam Tilahun) until consensus was reached. Articles identified through the electronic literature searches and from other sources were comprehensively reviewed based on the eligibility criteria for inclusion. The methods for data collection and analysis were based on the Cochrane Handbook of Systematic Reviews for Interventions [19].

\section{Quality assessment (risk of bias) in individual studies}

Two authors (Zeleke Abebaw and Kashaun Dessie) independently assessed the risk of bias for each included study by using the Cochrane Collaboration Risk of Bias Tool [19]. Articles were scored as having low, high, or unclear risk of bias for the seven attributes: sequence allocation, allocation concealment, blinding of participant and personnel, blinding of outcome assessor, incomplete outcome data, selective reporting, and other sources of bias. The two authors resolved disagreements in the assessment of risk of bias by discussion and consensus, consulting a third author (Binyam Tilahun) for any persistent disagreements. The kappa statistic was used to

Table 1 Search strategy for PubMed

$\overline{\left(\left(\left(\left(\left(\left(C_{\text {Child*}}[\mathrm{MeSH} \text { Terms] }) \text { OR Child* [All Fields]) OR Infant [MeSH Terms]) }\right.\right.\right.\right.\right.\right.}$ OR Infant [All fields])) AND

(((((((Text messag* [MeSH Terms]) OR Text messag* [All Fields]) OR Telemedicine [MeSH Terms]) OR mhealth [All fields]) OR Reminder systems [MeSH Terms]) OR Reminder systems [All Fields]) OR Messag* [All Fields])) AND

(((()(((Immuni* [MeSH Terms]) OR Immuni* [All Fields]) OR vaccin* [MeSH Terms]) OR vaccin* [All Fields]) OR "Mass Vaccination" [Mesh

Terms]) OR "Immunization Programs" [Mesh Terms]) OR "Immunization Schedule" [Mesh Terms]) OR "Immunization, Secondary" [Mesh Terms]) OR "Immunization, Passive" [Mesh Terms])) assess the level of agreement during risk of bias assessment by the two authors.

\section{Data extraction and management}

Data were extracted by two authors (Zeleke Abebaw and Kasahun Dessie) using a standardized data extraction format. The two authors' resolved disagreements by discussion consulting a third author (Binyam Tilahun) for any persistent disagreements. The final data were entered into the Cochrane Collaboration Review Manager Version 5.3 statistical software for risk of bias analysis. Finally, Stata version 14 software was used to conduct the meta-analysis.

\section{Assessment of heterogeneity}

Heterogeneity in meta-analysis refers to the variation in study outcomes between studies where $I^{2}$ statistic describes the percentage of variation across studies that is due to heterogeneity rather than chance [20]. We quantified the level of statistical heterogeneity with $I^{2}$ of $50 \%$ or more indicating the presence of substantial heterogeneity which is an indication to use random effects model and to perform subgroup analysis or metaregression [20]. We reviewed clinical heterogeneity in the setting, participants, intervention, and outcomes of included studies in order to make a qualitative assessment of the extent to which the included studies were similar to each other. Forest plot was used visually to assess the level of heterogeneity. In addition, Cochrane's $Q$ statistic was performed with a $P$ value for the chisquared test of less than 0.1 indicating the presence of statistical heterogeneity.

\section{Assessment of publication bias}

Publication bias occurs when the results of published studies are systematically different from the results of unpublished studies. In this review, evidence of publication bias was investigated by examining the symmetry of the funnel plot and by performing a Harbord statistical test [20].

\section{Measures of effect and reporting}

Systematic reviews and meta-analyses are essential tools for summarizing evidence for which PRISMA statement flow diagrams ensure transparent and complete reporting [21]. Forest plots and funnel plots were used to visualize the level of heterogeneity and publication bias, respectively. Random effects model was used during analysis, and risk ratios with their 95\% CI were used to present the pooled effect sizes [20]. 


\section{Assessment of certainty of evidence}

We assessed the certainty of evidence using GRADE (Grading of Recommendations, Assessment, Development and Evaluation). This method results in an assessment of the quality of the body of evidence as high, moderate, low, or very low. Body of the evidence was assessed against the following criteria: risk of bias, heterogeneity, imprecision, indirectness, and publication bias [19].

\section{Results}

\section{Selection of studies}

A total of 1771 articles were searched. Out of those 1771 articles, 558 duplicated articles were removed using Endnote7 software. Finally, the 1213 articles were screened based on the eligibility criteria. After screening of titles and abstracts, 28 articles were included for fulltext screening based on the eligibility criteria (Fig. 1). Finally, 10 studies were eligible and included for the review [22-31], while the remaining 18 full-text articles were excluded with reasons (Table 3 in the Appendix).

\section{Characteristics of included studies}

A total of ten articles met the inclusion criteria. These studies were conducted in the USA (5), Kenya
(2), Nigeria (1), Guatemala (1), and Zimbabwe (1) (Table 2). Author, year of publication, country, study design, study setting, population, intervention used, duration of intervention, timing of message, vaccine type, and outcome data were extracted to describe the characteristics of the studies. The total sample size of the studies was 10,625 (5273 in the intervention group and 5325 in the control groups). Majority $(60 \%)$ of the studies enrolled parents/women from health facilities [22, 23, 26, 29-31], three enrolled children who presented for a first dose [24, 25, 28], and one study enrolled caregivers at the village level [27].

Of the included studies, five (50\%) were published after 2015. The studies were mostly conducted in the USA, 5 (50\%), followed by Kenya, 2 (20\%). The follow-up period between studies varied from 14 weeks to 2 years with very high lost to follow-up in two studies $[22,30]$. The frequency of messages and timing of the text message reminders was different in the included studies where two studies [23, 25] sent the reminders three times for a single schedule. Four studies [24, 25, 27, 30] measured full vaccination coverage, while three studies measured the

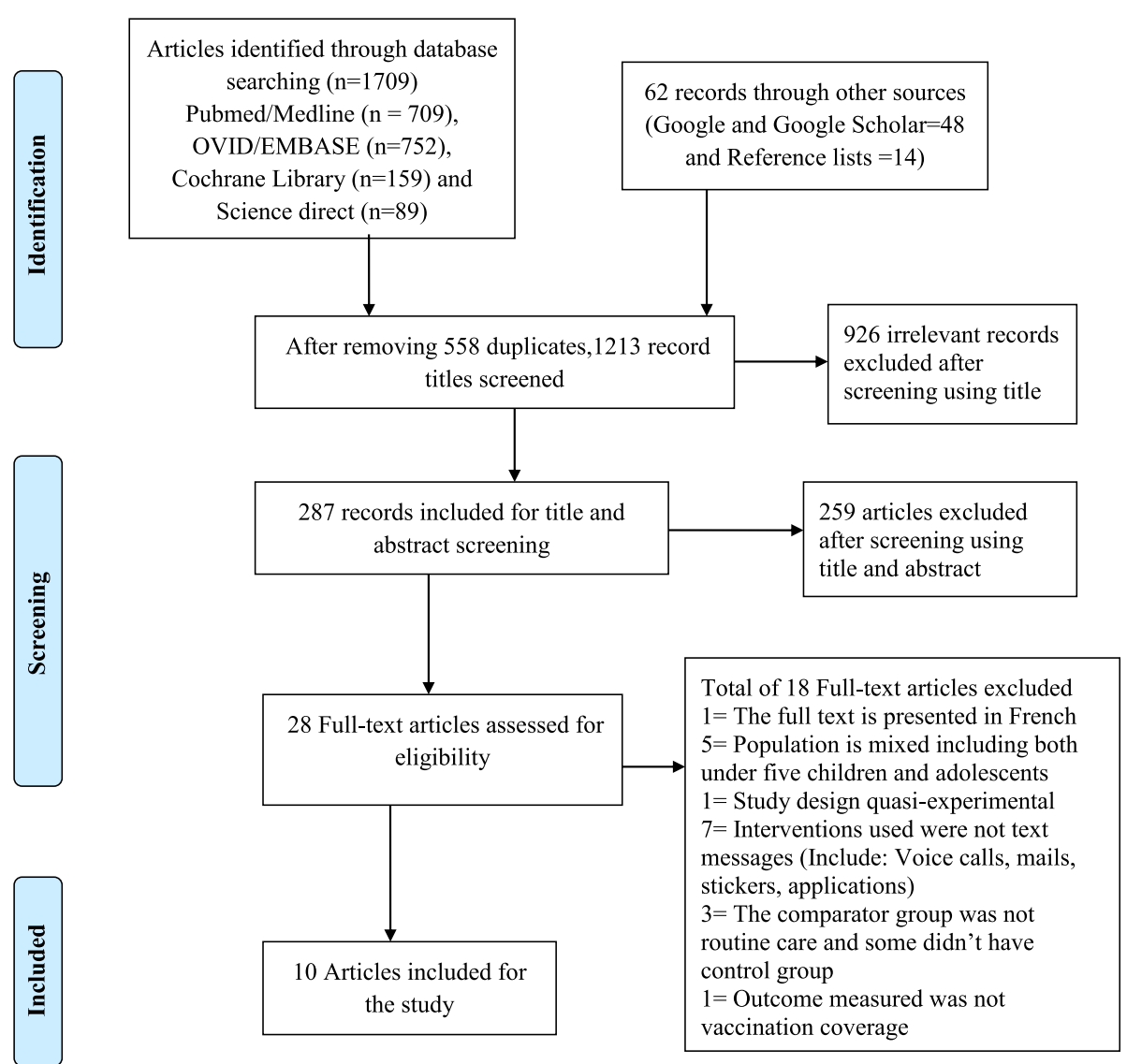

Fig. 1 PRISMA flow diagram representing the study selection process [21] 


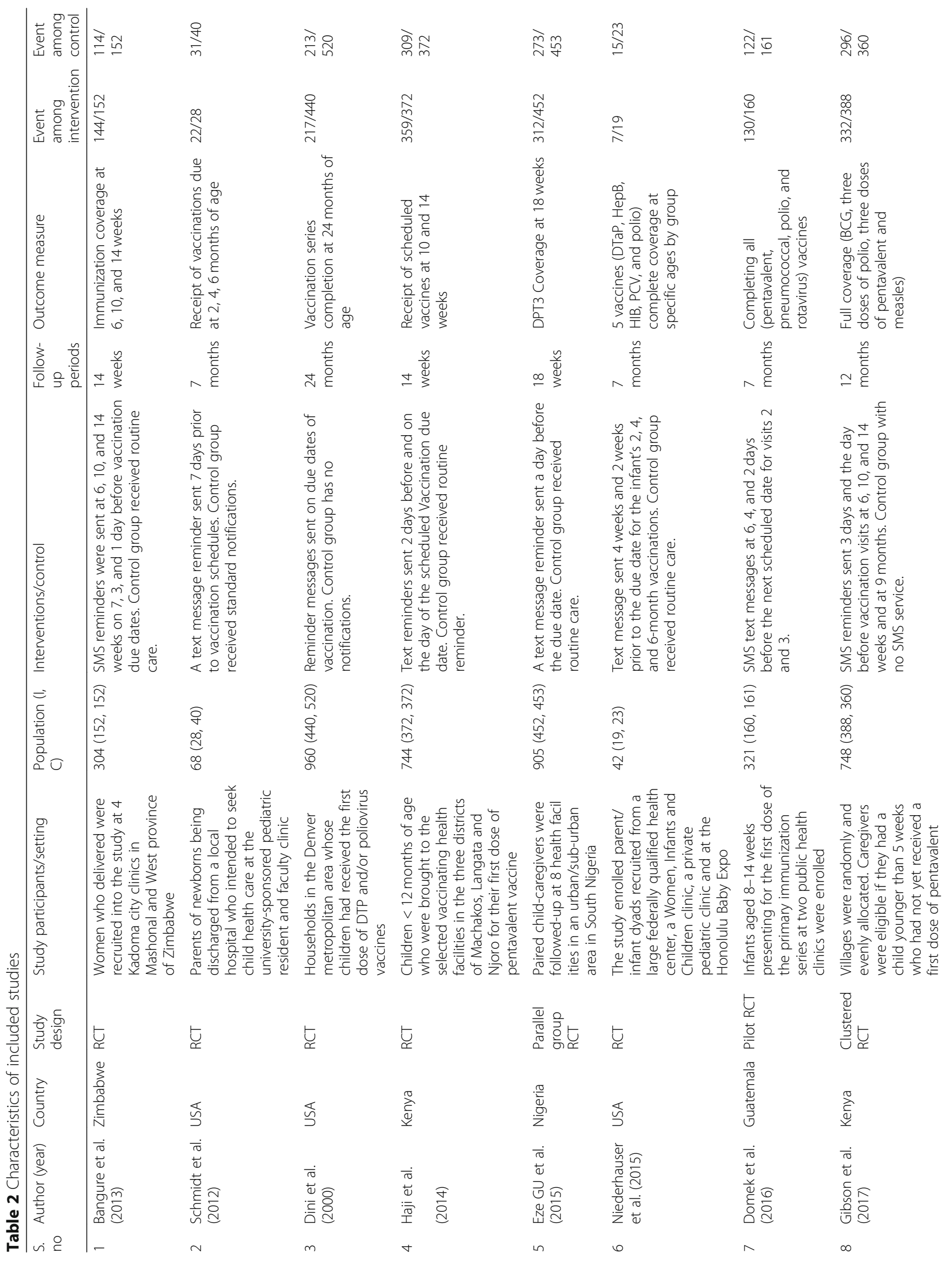




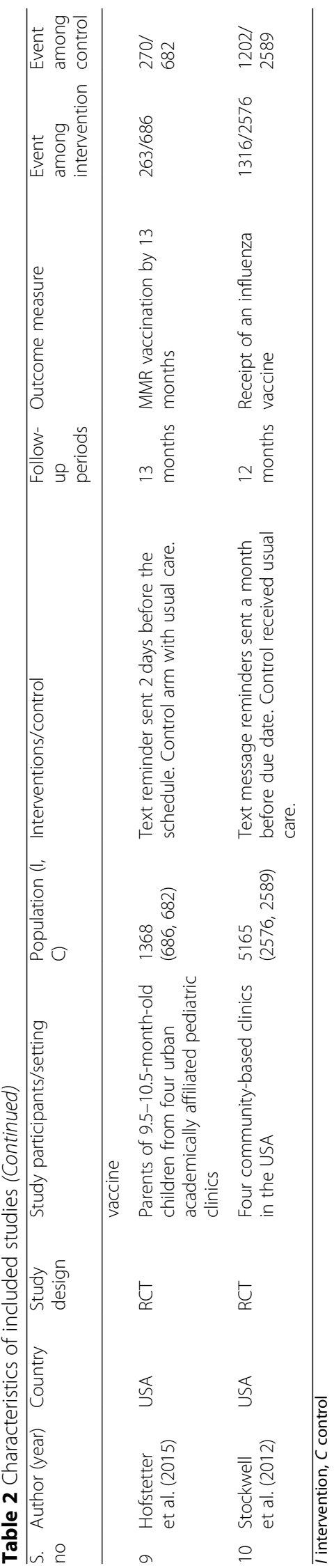


outcome for three doses of coverage [22, 23, 26]. The remaining studies measured the outcome at two doses [28] or one dose [29, 31] (Table 2).

\section{Quality (risk of bias) assessment for the included studies}

The risk of random sequence generation and allocation concealment was low for $80 \%$ of studies while unclear for the remaining two studies [24, 28]. Risk of bias in relation to blinding of participant and personnel was high for two studies [27, 29], while risk of bias in blinding of outcome assessments was low for majority of the studies. The risk of attrition bias was high for three studies $[22,29,30]$ and low risk for the remaining studies. The risk of selective reporting was high for one study [29] and low for the remaining studies. The kappa statistic showed that level of agreement during risk of bias assessment by the two authors (Zeleke Abebaw and Kasahun Dessie) was 69.2\% $(P$ value $<0.0001)$ (Fig. 3 in the Appendix).

\section{Quantitative data synthesis}

\section{Uptake of vaccination in all included studies}

The meta-analysis findings showed that there is a moderate level of statistically significant heterogeneity $\left(I^{2}=\right.$ $64.3 \% ; P=0.003)$ (Fig. 2). Since the $I^{2}(64.3 \%)$ was greater than $50 \%$, random effects model was used to estimate the pooled estimates. Accordingly, the meta-analysis revealed that there is a significant effect of text message reminders on childhood vaccination coverage $(\mathrm{RR}=1.11 ; 95 \% \mathrm{Cl}$ 1.05-1.17).

The predictive interval in this review overlaps the null $(0.95,1.29)$, indicating considerable uncertainty about the size and direction of an effect in a new study (Fig. 2).

\section{Uptake of vaccination by subgroups}

The magnitude of the heterogeneity (64.3\%) also showed that there is a need to conduct subgroup analysis to investigate the sources of heterogeneity.

\section{Subgroup analysis results by country income status}

In studies from high-income countries, the effect of a text message on childhood vaccination was positive and statistically insignificant $(\mathrm{RR}=1.06 ; 95 \% \mathrm{CI}$ $0.96-1.18)$ with moderate heterogeneity $\left(I^{2}=56.6 \%\right)$. The predictive interval in this review overlaps the null $(0.78,1.45)$, indicating considerable uncertainty about the size and direction of an effect in a new study.

On the other hand, there was a statistically significant positive effect of text message reminders among LMICs $(\mathrm{RR}=1.13$; 95\% CI $1.06 ; 1.21)$ with moderate heterogeneity $\left(I^{2}=70.4 \%\right)$. The predictive interval in this review overlaps the null $(0.91,1.41)$, indicating

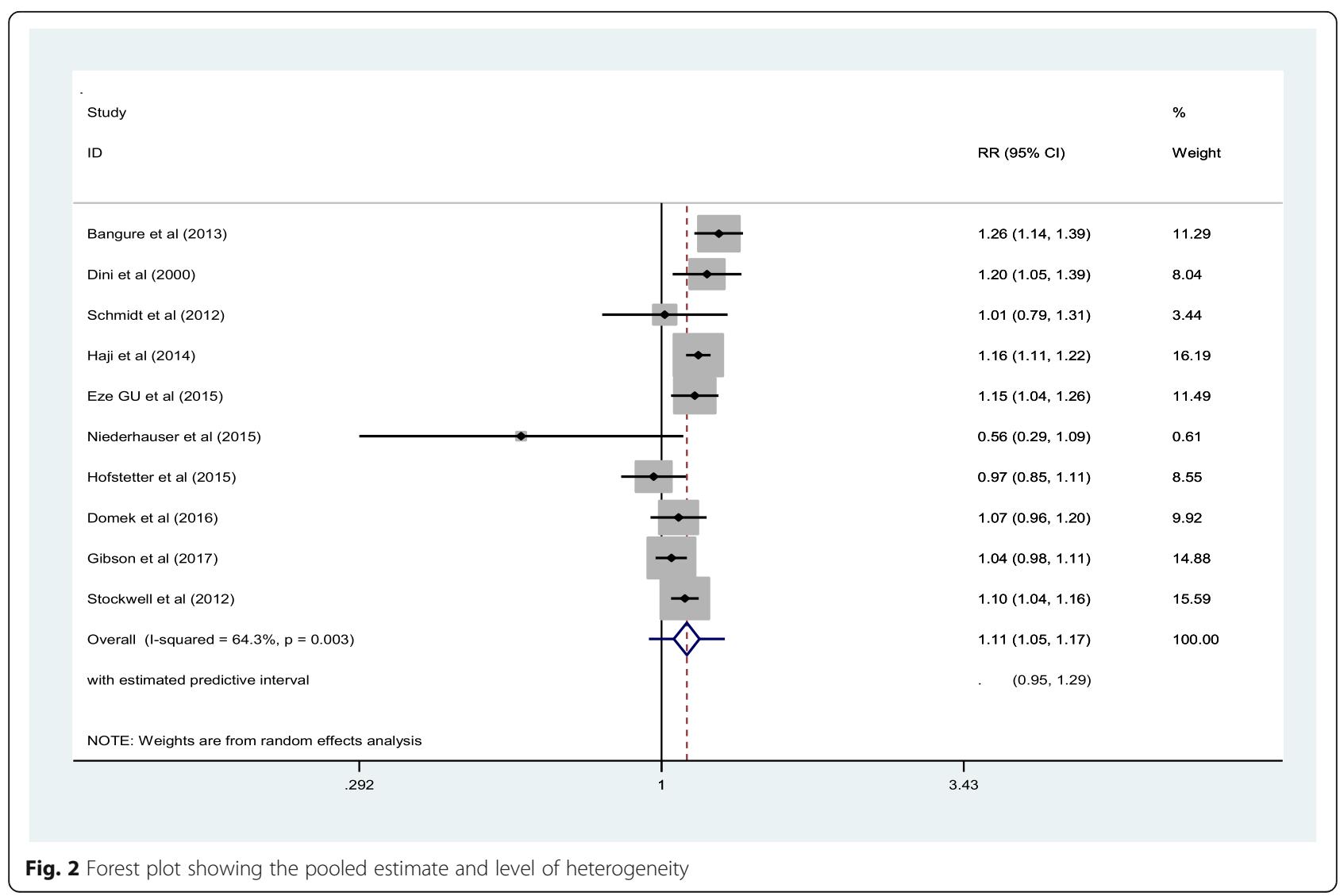


considerable uncertainty about the size and direction of an effect in a new study. The test for subgroup differences also indicated that there is no statistically significant difference across subgroups $(P=0.31)$ (Figure 4 in the Appendix).

\section{Subgroup analysis by timing of last message}

The forest plot showed that those studies in which the last reminder messages were sent on the due date had a statistically significant positive effect $(\mathrm{RR}=1.17 ; 95 \% \mathrm{CI}$ 1.11-1.22) on childhood vaccination with no heterogeneity $\left(I^{2}=0 \%\right)$. Similarly, sending reminder text messages 1 day before the due date also had a significant positive effect $(\mathrm{RR}=1.14 ; \quad 95 \%$ CI 1.02-1.28) on childhood immunization with substantial heterogeneity $\left(I^{2}=81.9 \%\right)$ . However, sending the text messages 2 or more days before the due date has no significant effect on childhood immunization coverage $(\mathrm{RR}=1.05,95 \%$ CI $0.96-1.13$ ) with low heterogeneity $\left(I^{2}=42 \%\right)$. The test for subgroup differences also indicated that there is no statistically significant difference across subgroups $(P=0.07)$ (Figure 5 in the Appendix).

\section{Subgroup analysis by number of messages for a single schedule}

The result showed that the number of messages sent for a given vaccination appointment schedule had different effects on childhood coverage. Accordingly, sending two text messages for one schedule visit had shown a statistically significant effect on childhood vaccination $(R R=$ 1.09; 95\% CI 1.01-1.18) with moderate heterogeneity $\left(I^{2}=74.4 \%\right)$. The test for subgroup differences also indicated that there is no statistically significant difference across subgroups $(P=0.76)$ (Figure 6 in the Appendix).

\section{Meta-regression}

Meta-regression is a tool used in meta-analysis to examine the impact of variables on the pooled effect size using regression-based techniques [20]. In this review, the effect of each individual study's follow-up time on the pooled estimate was assessed using meta-regression. Though not statistically significant, the findings revealed that an increase in a month of follow-up at which outcome was measured corresponds to a decrease in the $\operatorname{logRR}$ of intervention effect by $0.003(P$ value $=0.568)$ (Figure 7 in the Appendix).

\section{Assessment of publication bias}

A funnel plot provides evidence on the presence of small study effects based on subjective visual inspection. If the individual plots are located symmetrically in the funnel plot, it indicates the absence of publication bias [20]. In this review, the funnel plot observation suggests the absence of publication bias (Figure 8 in the Appendix).
To objectively measure the presence of publication bias, a Harbord test was also conducted. The results from the Harbord test suggested that there is no evidence for publication bias ( $P$ value, 0.340) (Figure 9 in the Appendix).

\section{Certainty of evidence}

The GRADE approach was used to assess the quality of evidence for systematic review and meta-analysis based on defined parameters as high, moderate, low, or very low [19]. In this review, the risk of bias was serious and there was moderate heterogeneity among studies making the quality of the body of evidence low level (Figure 10 in the Appendix).

\section{Discussion}

Multiple established and emerging strategies have been implemented to foster vaccination coverage globally. Despite the vast investment of resources in improving vaccination coverage in LMICs, few studies are available to inform policy and decision-making on childhood vaccination. In this review, ten articles were included to estimate the best available evidence on the effects of text message reminders in increasing vaccination coverage among children less than 5 years of age.

The pooled estimate revealed that there is a potential for text message reminders to improve childhood vaccination rates. However, the included studies showed moderate heterogeneity, which could result from clinical differences across studies. The precise effectiveness of these interventions is likely to be influenced by numerous factors such as country setting and nature of the interventions used. We did not find any important changes in the level of heterogeneity when we investigated the effect by country setting and number of text messages sent. However, the level of heterogeneity became lower in studies with text messages sent on the due date of vaccination with statistically significant positive effect.

Sending the last text message reminder nearer to the vaccination appointment date was also found to be more important since tackling forgetfulness needs more recent information with immediate actions.

We found relatively lower effectiveness of text reminders in high-income countries, despite the much greater level of economic advancement and immunization infrastructure in these areas than low- and middle-income countries. Differences in study settings could explain some of the observed differences. For example, studies done in the USA were more likely to include participants who presented to healthcare facilities and thus had enough resources for healthcare access (facility-based studies). 
We used the GRADE approach to assess the level of confidence to be placed on the evidence for the effects of text message reminders on vaccination coverage [19]. Randomized trials without important limitations provide high-quality evidence. However, in three of the included studies, the risk of attrition bias was high and in two studies the risk of blinding of personnel and participant was also high (Figure 10 in the Appendix). This led us to downgrade the quality of the evidence from high to moderate [19]. In addition, the presence of moderate heterogeneity further downgrades the quality of the evidence to a low level. Low quality of evidence implies the likely effect of text message reminders on childhood vaccination coverage with low confidence on the estimates to be implemented globally.

The findings from our review are in line with the findings of other reviews done on vaccination uptakes among adolescent and pregnant women where text messages are found to be effective reminders [13, 32, 33]. Stockwell et al. also presented a literature review of a broad range of health information technologies to improve vaccine communication and coverage and suggested that SMS is effective in improving vaccination uptake with the potential to penetrate large populations and relatively low cost [12]. Text messaging was also shown to be effective in nonvaccination-related health conditions [34]. The WHO has also reported that mHealth technologies are promising to strengthen health systems [35].

\section{Strengths and limitations}

The main strength of the current review lies in our adherence to international standardized guidelines on the conduct and reporting of systematic reviews. We included studies only from peer-reviewed English-language journals, which may have restricted our findings.

\section{Conclusions}

Mobile phone text message reminders have a potential to improve childhood vaccination coverages. This review highlights the potential benefits to childhood vaccine uptake of incorporating text message reminders into the standard management of childhood immunizations especially in low- and middle-income countries. The frequency and timing of the text message reminders are crucial in determining the effectiveness of text message reminders on childhood vaccination.

\section{Implications for practice}

The evidence presented in this review shows promise for the use of text message reminders to strengthen immunization programs. Thus, text message reminders deserve more attention as a potential innovation to improve healthcare operations more particularly in developing countries, where the mobile phone penetration is growing exponentially.

\section{Implications for future research}

Current evidence around mHealth interventions to improve vaccination is of low quality and with few studies. Therefore, more research is needed in this area.

\section{Appendix}

Table 3 Excluded studies with reasons

\begin{tabular}{|c|c|c|}
\hline $\begin{array}{l}\text { S. } \\
\text { no. }\end{array}$ & Authors (year) & Reasons for exclusion \\
\hline 1 & Li Chen (2016) & $\begin{array}{l}\text { The intervention was EPI application and text } \\
\text { reminder sent for both groups }\end{array}$ \\
\hline 2 & $\begin{array}{l}\text { Wakadha et al. } \\
\text { (2013) }\end{array}$ & $\begin{array}{l}\text { It was a feasibility study with no comparison } \\
\text { group of standard care }\end{array}$ \\
\hline 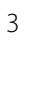 & $\begin{array}{l}\text { Uddin et al. } \\
\text { (2016) }\end{array}$ & $\begin{array}{l}\text { It was quasi-experimental study where the out } \\
\text { come was measured pre and post intervention } \\
\text { in both groups }\end{array}$ \\
\hline 4 & $\begin{array}{l}\text { Schlumberger } \\
\text { et al. (2015) }\end{array}$ & $\begin{array}{l}\text { The abstract was in English but full document } \\
\text { was in French. Though the author was } \\
\text { contacted but unable to get the English version } \\
\text { of the full text }\end{array}$ \\
\hline 5 & $\begin{array}{l}\text { Abramson et al. } \\
\text { (1995) }\end{array}$ & vere reminder cards and phone \\
\hline 6 & $\begin{array}{l}\text { Morgan et al. } \\
\text { (1998) }\end{array}$ & $\begin{array}{l}\text { Interventions were telephone call and mailed } \\
\text { reminders }\end{array}$ \\
\hline 7 & $\begin{array}{l}\text { Aragones et al. } \\
\text { (2015) }\end{array}$ & $\begin{array}{l}\text { Population includes children above } 5 \text { years of } \\
\text { age }\end{array}$ \\
\hline 8 & $\begin{array}{l}\text { Bjorr } \\
(199\end{array}$ & Inte \\
\hline 9 & $\begin{array}{l}\text { Kemp } \\
(2015\end{array}$ & $\begin{array}{l}\text { terventions mail } \\
s\end{array}$ \\
\hline 10 & $\begin{array}{l}\text { Stockwell et al. } \\
(2015)\end{array}$ & $\begin{array}{l}\text { Population extends above } 5 \text { years ( } 6 \text { months up } \\
\text { to } 8 \text { years) with no disaggregation for under- } \\
\text { five children }\end{array}$ \\
\hline 11 & $\begin{array}{l}\text { Zhou-Chen et al. } \\
\text { (2008) }\end{array}$ & $\begin{array}{l}\text { Outcome was improving attendance rates at a } \\
\text { health promotion center }\end{array}$ \\
\hline 12 & $\begin{array}{l}\text { Macknin et al. } \\
(2000)\end{array}$ & $\begin{array}{l}\text { Population extends beyond under } 5 \text { years } \\
(0-18 \text { years }) \text { and no separate data for children }\end{array}$ \\
\hline 13 & $\begin{array}{l}\text { Sthe } \\
(199\end{array}$ & roris were vorce Calis \\
\hline 14 & $\begin{array}{l}\text { Hofstetter et al. } \\
\text { (2015) }\end{array}$ & $\begin{array}{l}\text { Population beyond under } 5 \text { years ( } 6 \text { months to } \\
17 \text { years) with educational and interactive } \\
\text { messages }\end{array}$ \\
\hline 15 & $\begin{array}{l}\text { Lieu et al. } \\
\text { (1998) }\end{array}$ & $\begin{array}{l}\text { The study did not randomize patients to no } \\
\text { intervention group }\end{array}$ \\
\hline 16 & $\begin{array}{l}\text { Dombkowski } \\
\text { et al. (2014) }\end{array}$ & Intervention was mailed recall and reminder \\
\hline 17 & $\begin{array}{l}\text { O'Leary et al. } \\
\text { (2015) }\end{array}$ & $\begin{array}{l}\text { Population was adolescents without under-five } \\
\text { children }\end{array}$ \\
\hline 18 & $\begin{array}{l}\text { Irigoyen et al. } \\
\text { (2000) }\end{array}$ & $\begin{array}{l}\text { Interventions were phone calls and post card } \\
\text { reminders }\end{array}$ \\
\hline
\end{tabular}



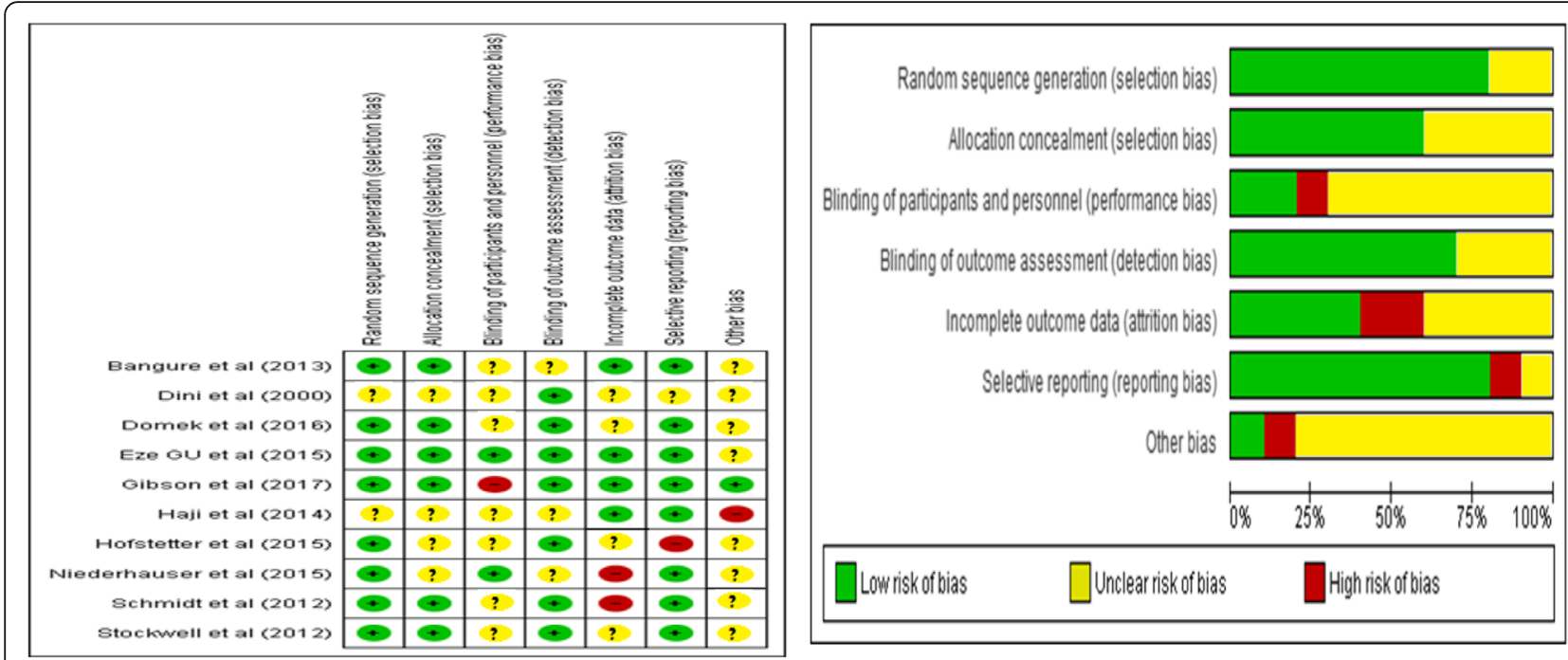

Fig. 3 Risk of bias assessment for the included studies

\section{Study}

LMIC

Bangure et al (2013)

Haji et al (2014)

Eze GU et al (2015)

Gibson et al (2017)

Subtotal (I-squared $=70.4 \%, p=0.009$ )

with estimated predictive interval

HIC

Dini et al (2000)

Schmidt et al (2012)

Niederhauser et al (2015)

Hofstetter et al (2015)

Stockwell et al (2012)

Subtotal ( 1 -squared $=56.6 \%, p=0.056$ )

with estimated predictive interval

Overall ( 1 -squared $=64.3 \%, p=0.003$ )

with estimated predictive interval

NOTE: Weights are from random effects analysis

$$
292
$$

RR (95\% C1)

$1.26(1.14,1.39)$

$1.16(1.11,1.22)$

$1.15(1.04,1.28)$

$1.07(0.96,120)$

$1.04(0.98,1.11)$

$1.13(1.06,1.21)$

(0.91, 1.41)

$1.20(1.05,1.39)$

$1.01(0.79,1.31)$

$0.56(0.29,1.09)$

$0.97(0.85,1.11)$

$1.10(1.04,1.16)$

$1.06(0.96,1.18)$

$(0.78,1.45)$

$1.11(1.05,1.17)$
$(0.95,1.29)$
$\%$

Weight

11.29

16.19

11.49
9.92

14.88

14.88
63.77

8.04
3.44

3.44
0.61

8.55

15.59
36.23

100.00

Fig. 4 Subgroup analysis by countries' income status

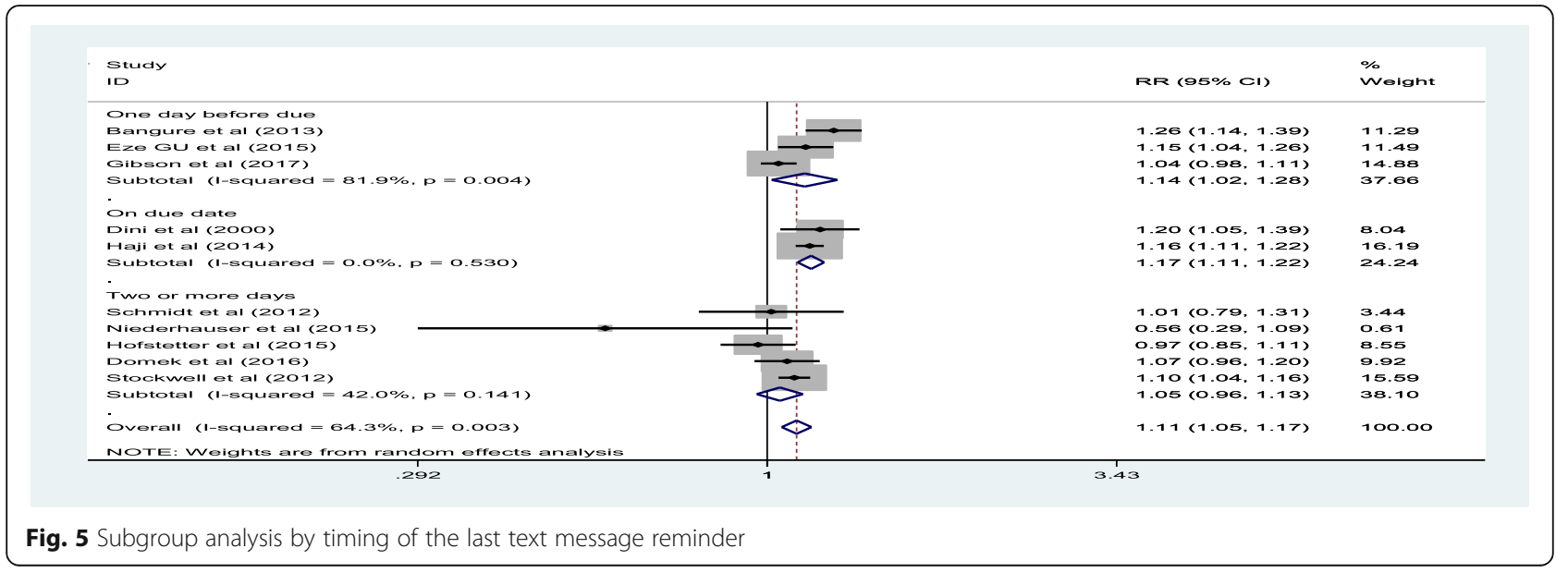




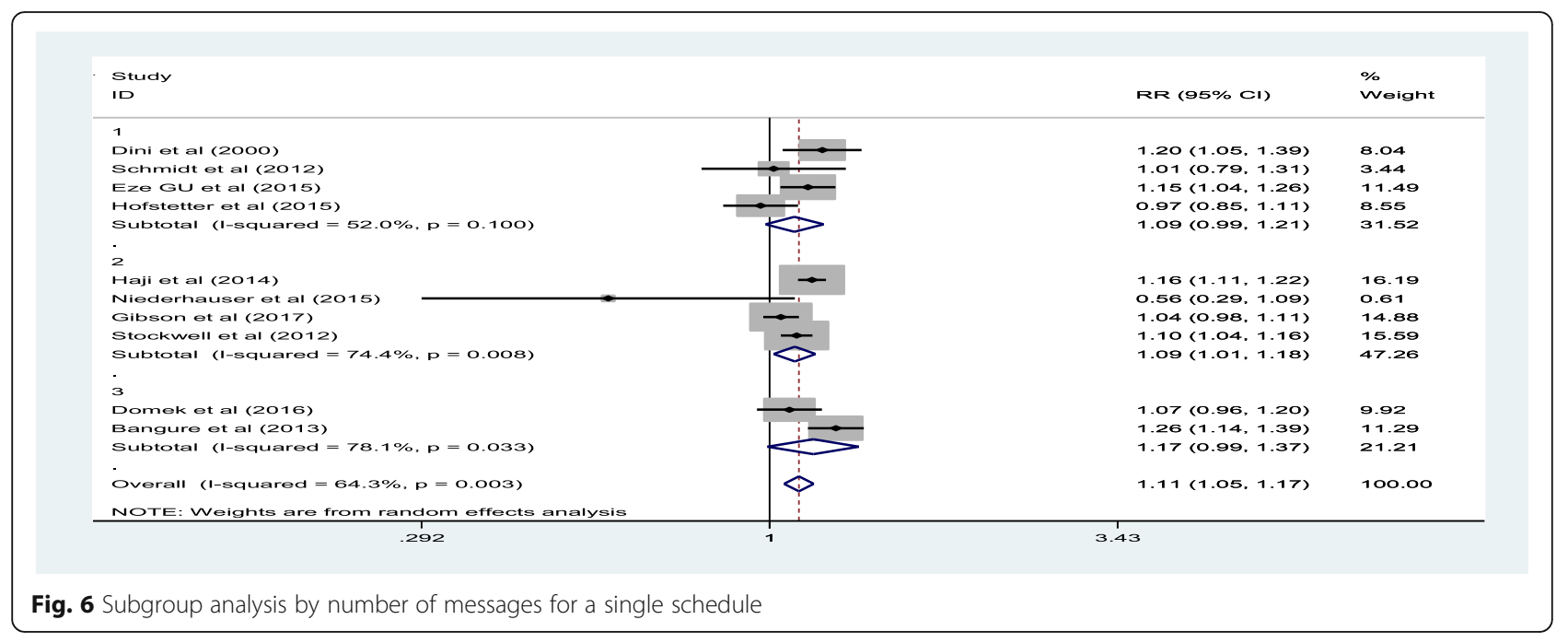

\begin{tabular}{|c|c|c|c|c|c|c|}
\hline \multicolumn{3}{|c|}{$\begin{array}{l}\text { - metareg logRR followuperiodmonths, wsse C SH } \\
\text { Meta-regression } \\
\text { REML estimate of between-study variance } \\
\text { t residual variation due to heterogeneity } \\
\text { Proportion of between-study variance explained } \\
\text { With Knapp-Hartung modification }\end{array}$} & $\operatorname{logRR}$ & \multicolumn{3}{|c|}{$\begin{array}{llr}\text { Number of obs } & = & 10 \\
\text { tauz } & = & 003159 \\
\text { I-squared_res } & = & 59.778 \\
\text { Adj R-squared } & = & 6.838\end{array}$} \\
\hline logRR & coef. & std. Err. & t & $P>|t|$ & [95t conf. & Intervalu \\
\hline $\begin{array}{r}\text { followupperiodmonths } \\
\text {-cons }\end{array}$ & $\begin{array}{r}-.0032362 \\
.1336584\end{array}$ & $\begin{array}{l}.0054295 \\
.0586242\end{array}$ & $\begin{array}{r}-0.60 \\
2.28\end{array}$ & $\begin{array}{l}0.568 \\
0.052\end{array}$ & $\begin{array}{l}-.0157566 \\
-.0015291\end{array}$ & $\begin{array}{r}.0092843 \\
.268846\end{array}$ \\
\hline \multicolumn{7}{|c|}{$\begin{array}{l}\text { end of do-file } \\
\text { Fig. } 7 \text { Outputs of meta-regression analysis }\end{array}$} \\
\hline
\end{tabular}

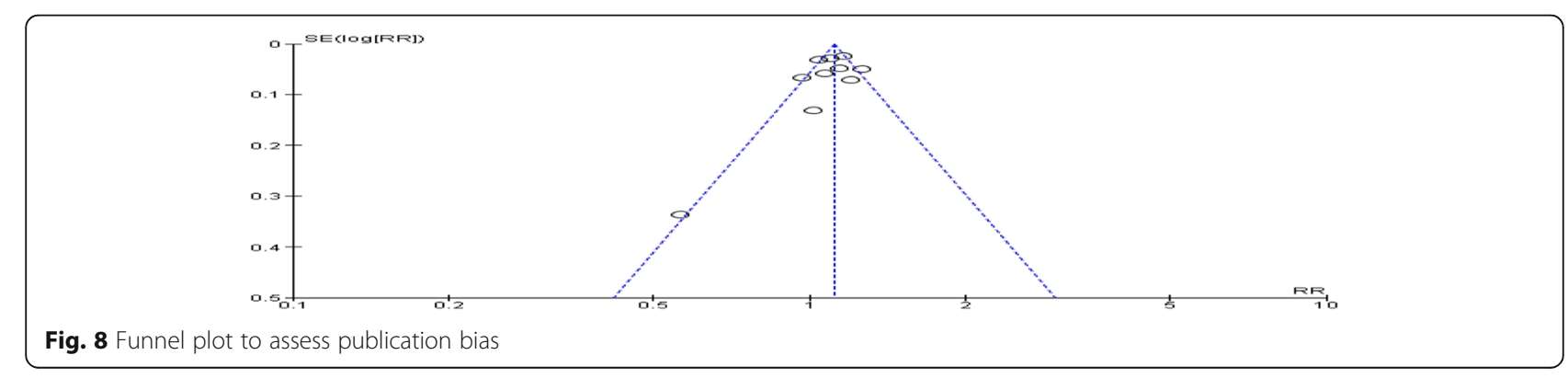




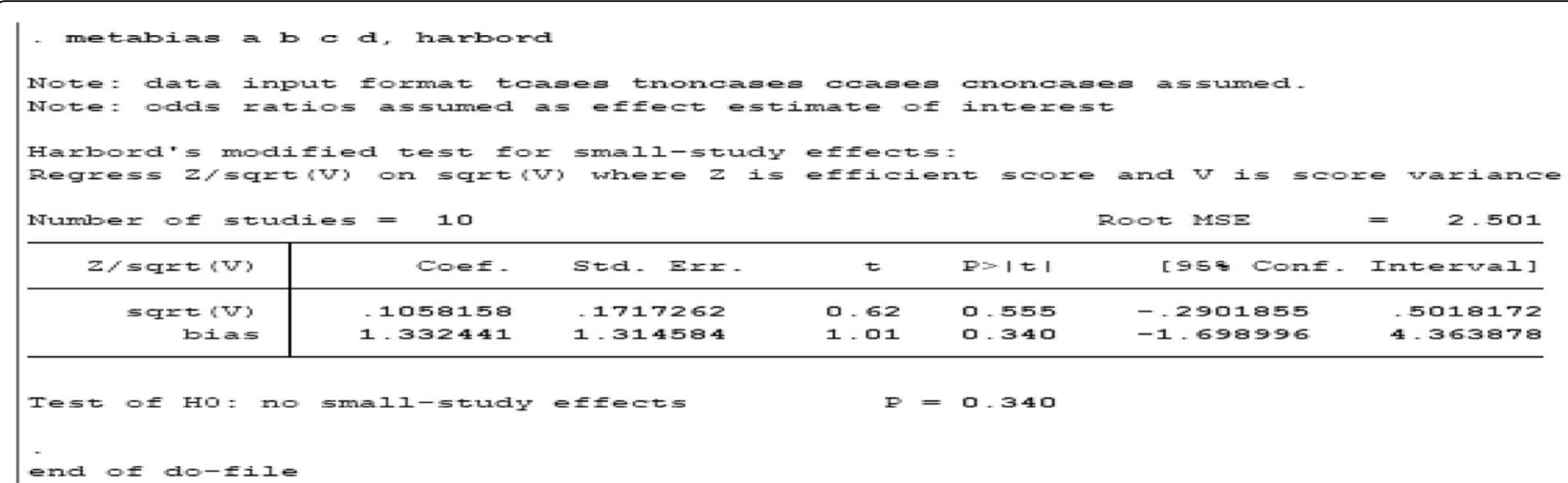

Fig. 9 Outputs of the Harbord test to assess publication bias

\begin{tabular}{|c|c|c|c|c|c|c|c|c|c|c|c|c|c|}
\hline \begin{tabular}{|l|l|l|} 
GRADEpro & GDT \\
\end{tabular} & \multicolumn{10}{|c|}{$\nabla$ mHealth } & 40 & \multicolumn{2}{|c|}{ zabebe@yahoo.com } \\
\hline & \multicolumn{8}{|c|}{ - Should Text Message reminders vs. Standard care be used for childhood vaccination? } & \multicolumn{3}{|c|}{ Bottom panel * Explanations } & - Help & In \\
\hline (1) Tasks: & \multicolumn{13}{|c|}{ Text Message reminders compared to Standard care for childhood vaccination } \\
\hline 88 Team & \multicolumn{7}{|c|}{ Quality assessment } & \multicolumn{5}{|c|}{ Summary of findings } & \multirow[b]{2}{*}{ Importance } \\
\hline$\$$ Scope & $\begin{array}{l}\text { № of } \\
\text { studies }\end{array}$ & Study design & Risk of bias & $\begin{array}{c}\text { Inconsistenc } \\
y\end{array}$ & Indirectness & Imprecision & Other considerations & $\begin{array}{l}\text { No of } p \text { a } \\
\text { Text Message } \\
\text { reminders }\end{array}$ & $\begin{array}{l}\text { patients } \\
\text { Standard care }\end{array}$ & $\begin{array}{l}\text { Ef } \\
\text { Relative } \\
\text { (95\% Cl) }\end{array}$ & $\begin{array}{l}\text { :ffect } \\
\text { Absolute } \\
(95 \% \text { CI) }\end{array}$ & Quality & \\
\hline [1] References & \multicolumn{13}{|c|}{ Childhood immunization } \\
\hline If Comparisons & 10 & $\begin{array}{l}\text { randomised } \\
\text { trials }\end{array}$ & serious $^{\mathrm{a}}$ & serious $^{b}$ & not serious & not serious & none & $\begin{array}{l}3102 / 5273 \\
(58.8 \%)\end{array}$ & $\begin{array}{l}2845 / 5352 \\
(53.2 \%)\end{array}$ & $\begin{array}{l}\text { RR } 1.11 \\
\text { (1.05 to } 1.17)\end{array}$ & $\begin{array}{l}58 \text { more per } \\
1,000 \\
\text { (from } 27 \text { more } \\
\text { to } 90 \text { more) }\end{array}$ & $\begin{array}{c}\oplus \oplus 00 \\
\text { LOW }\end{array}$ & CRITICAL \\
\hline Evidence table & \multicolumn{7}{|c|}{ Add outcome } & \multicolumn{6}{|c|}{ Import outcome(s) } \\
\hline
\end{tabular}

Fig. 10 Assessment of certainty of evidence using GRADEpro software 


\section{Abbreviations}

Cl: Confidence interval; GRADE: Grading of Recommendations, Assessment, Development and Evaluation; MeSH: Medical Subject Headings; PRISMA: Preferred Reporting Items for Systematic Reviews and Meta-Analysis; RCT: Randomized controlled trial; RR: Relative risk; SRMA: Systematic review and meta-analysis; WHO: World Health Organization

\section{Acknowledgements}

The authors thank the University of Gondar and all the authors of the original articles.

\section{Authors' contributions}

ZAM conceived and designed the review and did the article searching, critical appraisal, data extraction, data analysis, interpretation of results, and write up of the manuscript. BCT and KAG were involved in the study design, critical appraisal, interpretation of results, and review of the manuscript. KDG did the article searching, critical appraisal, data extraction, data analysis, interpretation of results, and review of the manuscript. MCW provided critical appraisal and reviewed and edited the manuscript. All authors read and approved the manuscript.

\section{Funding}

There is no organizational funding for this systematic review and metaanalysis.

\section{Availability of data and materials}

The extracted data is available upon request.

\section{Ethics approval and consent to participate}

Not applicable.

\section{Consent for publication}

Not applicable.

\section{Competing interests}

The authors declare that they have no competing interests.

\section{Author details}

'Department of Health Informatics, Institute of Public Health, College of Medicine and Health Sciences, University of Gondar, Gondar, Ethiopia. ${ }^{2}$ Department of Epidemiology and Biostatistics, Institute of Public Health, College of Medicine and Health Sciences, University of Gondar, Gondar, Ethiopia. ${ }^{3}$ Vanderbilt University Medical Center, Nashville, TN 37232, USA.

\section{Received: 4 January 2018 Accepted: 26 May 2019}

\section{Published online: 28 June 2019}

\section{References}

1. WHO. Immunization Coverage fact sheet. 2016. URL: http://www.who.int/ mediacentre/factsheets/fs378/en/ accessed on September 15, 2017.

2. Machingaidze S, Wiysonge CS, Hussey GD. Strengthening the expanded programme on immunization in Africa: looking beyond 2015. PLoS Med. 2013;10(3):e1001405

3. Jani CDS, Jani I, Bjune G. Risk factors for incomplete vaccination and missed opportunity for immunization in rural Mozambique. BMC Public Health. 2008;8(1):161.

4. Adedokun ST, Uthman OA, Adekanmbi VT, Wiysonge CS. Incomplete childhood immunization in Nigeria: a multilevel analysis of individual and contextual factors. BMC Public Health. 2017;17(1):236.

5. Debie A, Taye B. Assessment of fully vaccination coverage and associated factors among children aged 12-23 months in Mecha District, north west Ethiopia: a cross-sectional study. Sci J Public Health. 2014;2(4):342-8.

6. Patel T, Pandit N. Why infants miss vaccination during routine immunization sessions? Study in a rural area of Anand District, Gujarat. Indian J Public Health. 2011:55(4):321.

7. Falagas ME, Zarkadoulia E. Factors associated with suboptimal compliance to vaccinations in children in developed countries: a systematic review. Curr Med Res Opin. 2008;24(6):1719-41.

8. Abahussin AA, Albarrak Al. Vaccination adherence: review and proposed model. J Infect Public Health. 2016;9(6):781-9.
9. Kay M, Santos J, Takane M. mHealth: new horizons for health through mobile technologies. World Health Organization. 2011;64(7):66-71.

10. Labrique AB, Vasudevan L, Kochi E, Fabricant R, Mehl G. mHealth innovations as health system strengthening tools: 12 common applications and a visual framework. Global Health Sci Pract. 2013;1(2):160-71.

11. Kannisto A, Koivunen M, Välimäki M. Use of mobile phone text message reminders in health care services: a narrative literature review. J Med Internet Res. 2014;16(10).

12. Stockwell MS, Fiks AG. Utilizing health information technology to improve vaccine communication and coverage. Human Vaccin Immunother. 2013; 9(8):1802-11.

13. Watterson J, Walsh J, Madeka I. Using mHealth to improve usage of antenatal care, postnatal care, and immunization: a systematic review of the literature. Biomed Res Int. 2015;2015.

14. Chachou MJ, Mukinda FK, Motaze V, Wiysonge CS. Electronic and postal reminders for improving immunisation coverage in children: protocol for a systematic review and meta-analysis. BMJ Open. 2015:5(10): e008310.

15. Aranda-Jan CB, Mohutsiwa-Dibe N, Loukanova S. Systematic review on what works, what does not work and why of implementation of mobile health (mHealth) projects in Africa. BMC Public Health. 2014;14(1):188.

16. HarveyH, N. Reissland and J. Mason. Parental reminder, recall and educational interventions to improve early childhood immunisation uptake: a systematic review and meta-analysis. Vaccine. 2015;33(25):2862-80.

17. Wakadha H, Chandir S, Were E, Rubin A, Obor D, Levine O, et al. The feasibility of using mobile-phone based SMS reminders and conditional cash transfers to improve timely immunization in rural Kenya. Vaccine. 2013. 31(6):987-93.

18. Restrepo M, Barros A, Wong K, Johnson H, Pariyo G, Wehrmeister F, Victora C. Missed opportunities in full immunization coverage: findings from lowand lower-middle-income countries. Glob Health Action. 2016;9(1):30963.

19. Higgins JPT, Green S. Cochrane handbook for systematic reviews of interventions; 2008.

20. Ahn EJ, Kang $\mathrm{H}$. Introduction to systematic review and meta-analysis. Korean J Anesthesiol. April 2018;71(2)

21. Liberati A, Altman DG, Tetzlaff J, Mulrow C, Gøtzsche PC, et al. The PRISMA statement for reporting systematic reviews and meta-analyses of studies that evaluate health care interventions: explanation and elaboration. PLoS Med. 2009;6(7):e1000100. https://doi.org/10.1371/journal.pmed.1000100.

22. Ahlers-Schmidt C, Chesser A, Nguyen T, Brannon J, Hart T, Williams K, et al. Feasibility of a randomized controlled trial to evaluate text reminders for immunization compliance in kids (TRICKs). Vaccine. 2012;30(36):5305-9.

23. Donewell B, Daniel C, Notion G, Tawanda M, Gibson M, Mufuta T, Lucia T. Effectiveness of short message services reminder on childhood immunization programme in Kadoma, Zimbabwe-a randomized controlled trial, 2013. BMC Public Health. 2015;15(1):137.

24. Dini E, Linkins R, Sigafoos J. The impact of computer-generated messages on childhood immunization coverage. Am J Prev Med. 2000;18(2):132-9.

25. Domek G, Contreras R, O'Leary S, Bull S, Furniss A, Kempe A, Asturias E. SMS text message reminders to improve infant vaccination coverage in Guatemala: a pilot randomized controlled trial. Vaccine. 2016;34(21):2437-43.

26. Eze $\mathrm{G}$, Adeleye $\mathrm{O}$. Enhancing routine immunization performance using innovative Technology in an Urban Area of Nigeria. West Afr J Med. 2015; 34(1):3-10.

27. Gibson D, Kagucia E, Ochieng B, Hariharan N, Obor D, Moulton L, et al. The mobile solutions for immunization (M-SIMU) trial: a protocol for a cluster randomized controlled trial that assesses the impact of mobile phone delivered reminders and travel subsidies to improve childhood immunization coverage rates and timeliness in western Kenya. JMIR research protocols. 2016;5(2)

28. Adam H, Lowther S, Ngan'ga Z, Gura Z, Tabu C, Sandhu H, et al. Reducing routine vaccination dropout rates: evaluating two interventions in three Kenyan districts, 2014. BMC Public Health. 2016;16(1):152.

29. Hofstetter A, DuRivage N, Vargas C, Camargo S, Vawdrey D, Fisher A, et al. Text message reminders for timely routine MMR vaccination: a randomized controlled trial. Vaccine. 2015;33(43):5741-6.

30. Niederhauser $V$, Johnson $M$, Tavakoli A. Vaccines4Kids: assessing the impact of text message reminders on immunization rates in infants. Vaccine. 2015; 33(26):2984-9.

31. Stockwell M, Kharbanda E, Martinez R, Vargas C, Vawdrey D, Camargo S. Effect of a text messaging intervention on influenza vaccination in an 
urban, low-income pediatric and adolescent population: a randomized controlled trial. JAMA. 2012;307(16):1702-8.

32. Odone A, Ferrari A, Spagnoli F, Visciarelli S, Shefer A, Pasquarella C, et al. Effectiveness of interventions that apply new media to improve vaccine uptake and vaccine coverage: a systematic review. Human Vaccin Immunother. 2015;11(1):72-82.

33. Sondaal S, Browne J, Coleman M, Borgstein A, Miltenburg A, Verwijs M, et al. Assessing the effect of mHealth interventions in improving maternal and neonatal care in low-and middle-income countries: a systematic review. PLoS One. 2016;11(5):e0154664.

34. Militello L, Kelly S, Melnyk B. Systematic review of text-messaging interventions to promote healthy behaviors in pediatric and adolescent populations: implications for clinical practice and research. Worldviews EvidBased Nurs. 2012;9(2):66-77.

35. WHO. mHealth New horizons for health through mobile technologies. In: Global observatory for eHealth series, vol. 3; 2011.

\section{Publisher's Note}

Springer Nature remains neutral with regard to jurisdictional claims in published maps and institutional affiliations.

Ready to submit your research? Choose BMC and benefit from:

- fast, convenient online submission

- thorough peer review by experienced researchers in your field

- rapid publication on acceptance

- support for research data, including large and complex data types

- gold Open Access which fosters wider collaboration and increased citations

- maximum visibility for your research: over $100 \mathrm{M}$ website views per year

At BMC, research is always in progress.

Learn more biomedcentral.com/submissions 